\title{
Experimental design of EDM pulse generator based on VB and SCM
}

\author{
Renjie $\mathrm{Ji}^{\mathrm{a}}$, Yonghong $\mathrm{Liu}^{\mathrm{b}}$, Xiaopeng $\mathrm{Li}^{\mathrm{c}}$ and Xin Dong ${ }^{\mathrm{d}}$
}

No.66, Changjiangxi Road, Economic and Technological Development Zone, Qingdao City

Shandong Province, China

ajirenjie202@yahoo.cn, bliuyh@upc.edu.cn, 'Ixp@upc.edu.cn, d1464610695@qq.com

Keywords: electrical discharge machining (EDM), pulse generator, VB, SCM

\begin{abstract}
Pulse generator is the key equipment of electrical discharge machining (EDM). Considering the disadvantage of the traditional EDM pulse generator, a new pulse generator based on VB and SCM is designed. The simulation and serial communication with SCM are achieved, and the pulse width, pulse period, and phase sequence difference can be adjusted by the upper computer at a wide range. The simulation experiment results show that the pulse parameter can be adjusted conveniently, and the effects of the pulse parameter on the process performance can be understood easily.
\end{abstract}

\section{Introduction}

Electrical discharge machining (EDM) is a kind of machining process that removes material utilizing electrical energy and thermal energy, and it has been rapidly developed due to its huge advantage in machining hard cutting material, special and complicated parts [1,2]. Pulse generator provides energy to the discharge gap to remove material, so it is the key equipment that directly influences the machining speed, machining precision, machining stability, surface roughness of the workpiece and electrode wear ratio in EDM $[3,4]$. The common pulse generator in EDM is the transistor pulse generator, and the pulse signal is produced by resistors and capacitors when they charge or discharge. However, the pulse signal can only be gradually adjusted, and the pulse width and pulse period cannot be adjusted independently. Thus, it is hard to adjust each pulse parameter accurately according to the EDM request.

The PWM signal, whose frequency, duty ratio and phase sequence difference can be adjusted, is produced by the AT89C52 SCM to drive the MOSFET in this paper. Furthermore, VB language is used to program the controlling interface of the pulse generator, the simulation and serial communication with SCM are achieved, and the pulse width, pulse period, and phase sequence difference can be randomly adjusted at a wide range. As a result, the pulse parameters can be adjusted conveniently during EDM, and the effects of the pulse parameter on the process performance can be understood easily.

\section{Production of PWM Signal by SCM}

Modular programming is used in the pulse generator in this paper, moreover, the assignment between modules is specific, the repeated design is avoided, and the relative independence between modules is built. The power system mainly contains two modules which are the PWM signal module and the serial communication module.

The PWM Signal Module. In this module, three channels of PWM signals are produced from the SCM timer interrupt, and they are exported by P10, P11, and P12 of the AT89C52 SCM. T0 is used as the timer, and the minimum resolution is $0.2 \mathrm{~ms}$. The program flow chart of the PWM signal module is shown in Fig. 1, and the pwm, mk, aq represent the serial sequence difference, the pulse width, and the pulse period, respectively, which are transferred from the upper computer to SCM. 


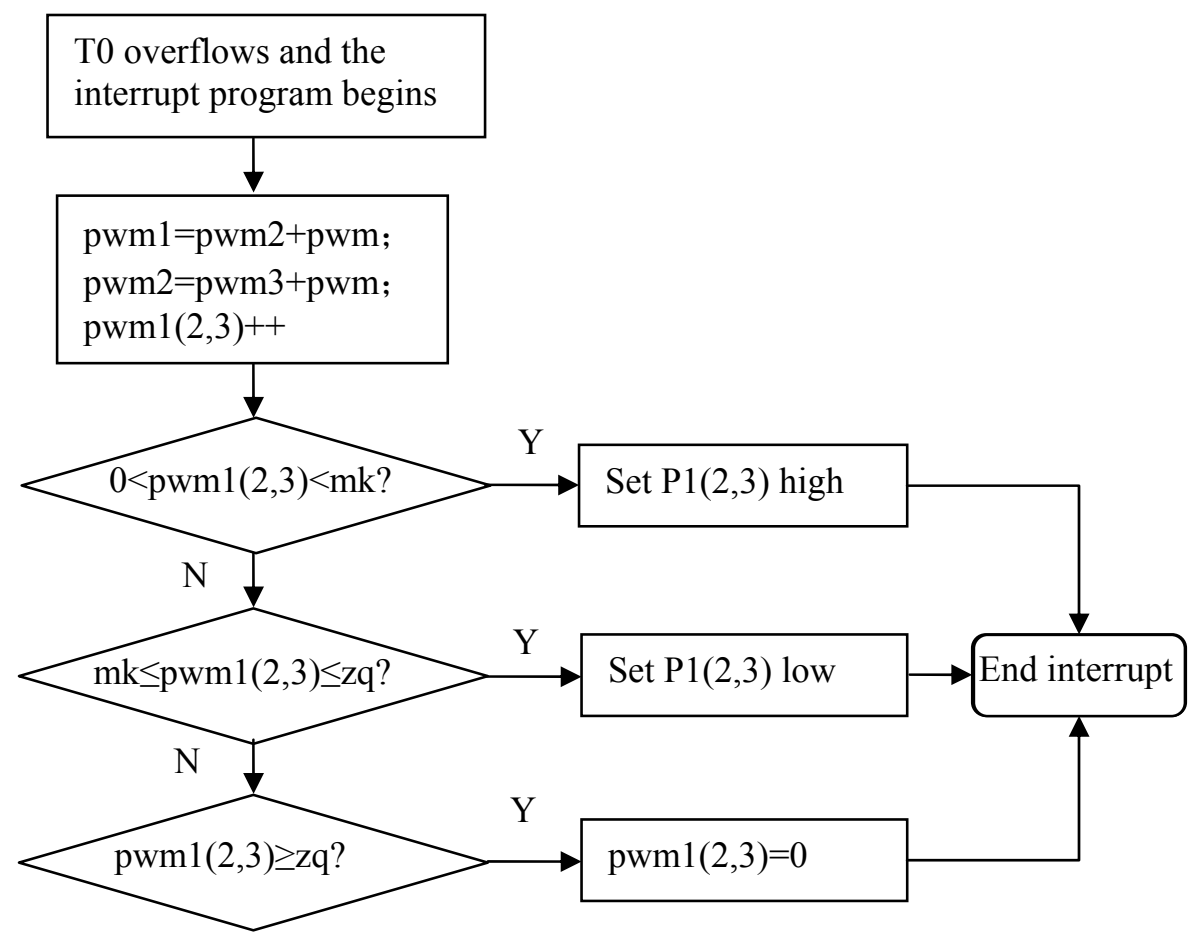

Fig. 1 The flow chart of the PWM signal module

The Serial Communication Module. The flow chart of the serial communication module is shown in Fig. 2. In this module, the data is received and transmitted by a full duplex UART asynchronous serial port of the AT89C52 SCM. This module is composed of the serial reception interruption subprogram. The timer $\mathrm{T} 1$ is used as the baud rate generator in the serial communication. The serial initialization subprogram and the serial reception interrupt subprogram are programmed.

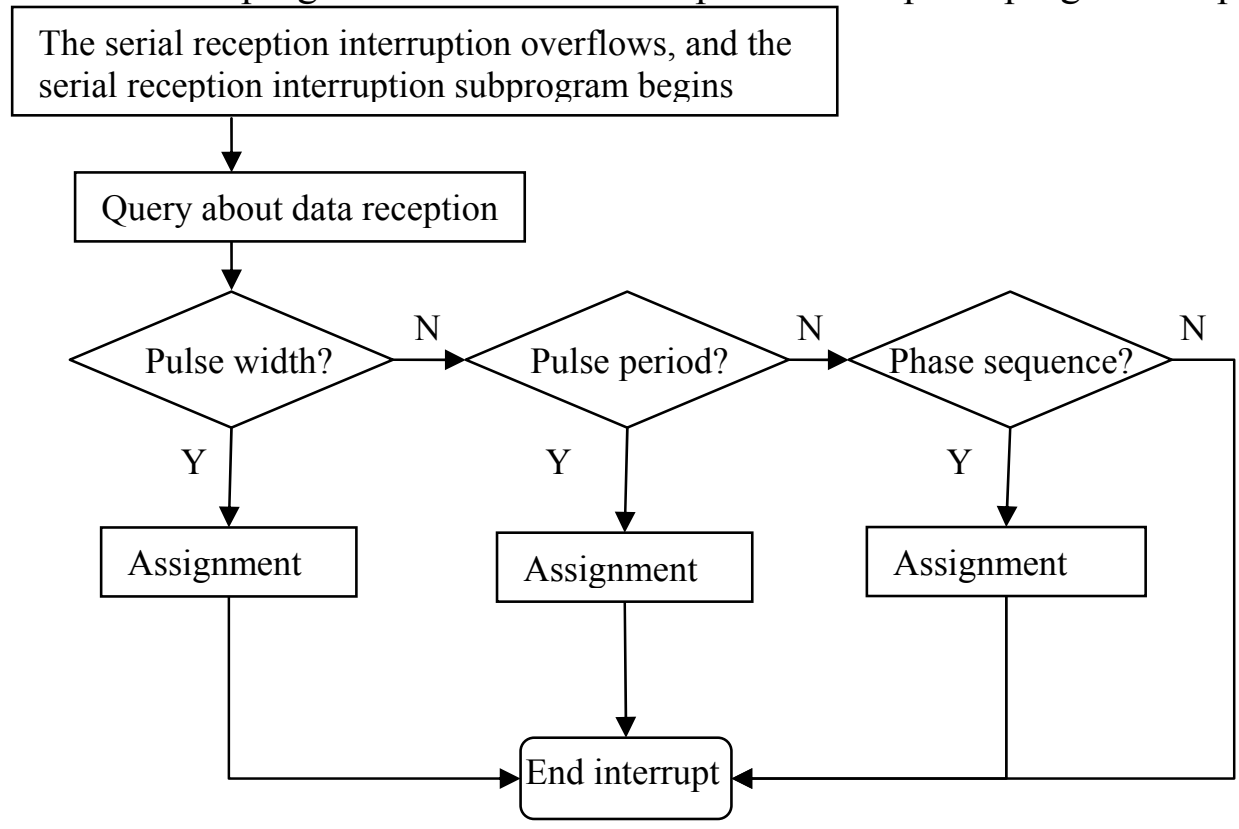

Fig. 2 The flow chart of the serial communication module

\section{Interface Design of EDM Pulse Generator Based on VB}

The interface of EDM pulse generator is programmed by VB language in this paper. The pulse width, pulse period, and phase sequence difference of the PWM signal can be adjusted with the serial communication between MSComm and SCM. The interface is shown in Fig. 3. It can be seen from this figure that the theoretical duty ratio and frequency can be computed, and the theoretical wave can 
be drawn. The interface mainly contains variable setting, variable adjustment, result show, serial communication, serial adjustment, etc.

The use of this interface is as follows: input the expected pulse width, pulse period and phase sequence difference whose unit is ms, in the 'Variable Setting' case; adjust the variable in the 'Variable Adjustment' case and select button 1 or 0.2 as the step; click the 'Compute' button, and the theoretical duty ratio and frequency would be shown in the 'Result Show' case; click the 'Plot' button, and the theoretical PWM wave would be shown in the 'Wave Display' case; set the baud rate, parity bit, data bit, stop bit and COM port in the 'SCM Communication' case; click the 'Open Serial Port' button, the serial port will show 'on', 'Data can be transmitted now' prompt case will occur; and the pulse width, pulse period and phase sequence difference can be transmitted and received in the 'Serial Communication' case.

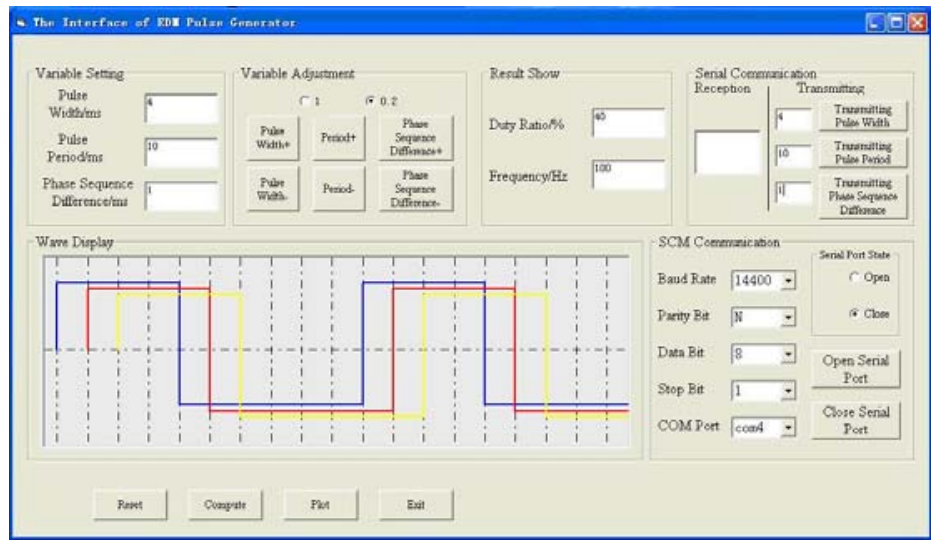

Fig. 3 The interface of EDM pulse generator based on VB

\section{The Simulation Experiment of the EDM Pulse Generator}

The EDM pulse generator uses the AT89C52 SCM as the core in this paper, and it is mainly composed of the pulse generator-control circuit, the opto-couple circuit and the power amplifier output circuit. The parameters of the pulse generator can be set and adjusted accurately by the upper computer.

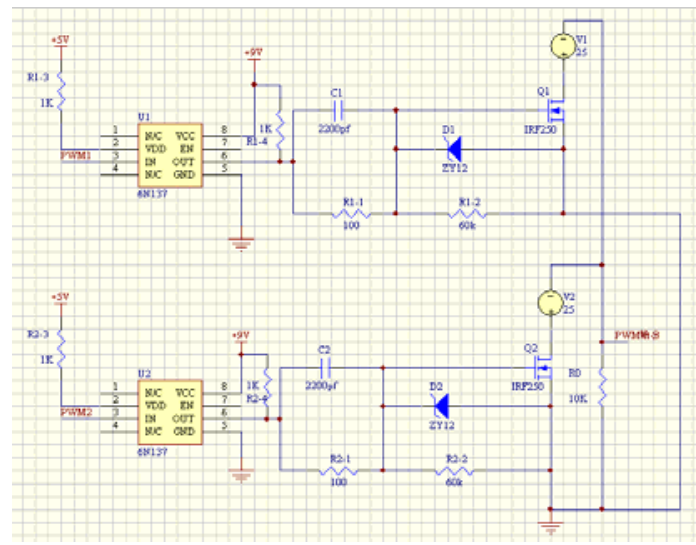

Fig. 4 Schematic control circuit diagram of the simulation experiment

The pulse width, pulse period, and phase sequence difference of the pulse generator are controlled by the SCM in the pulse generator-control circuit. However, the pulse generated by the SCM cannot control the MOSFET directly to generate power pulse, and a power amplifier is needed. In this paper, the optocoupler is used to achieve the optical isolation between the main control circuit and power amplifier circuit, which can effectively improve the anti-jamming capacity of the control system. The controllable PWM pulse signal generated by the SCM I/O port is amplified and used to control the on-off of the MOSFET. The control circuit is shown in Fig. 4. It can be seen from Fig. 4 that U1 and $\mathrm{U} 2$ are both the $6 \mathrm{~N} 137$ optocouplers, the third port is used to receive the PWM signal from the SCM, 
and the sixth port is used to export the signal. Q1 and Q2 are IRF250 which is a kind of fast on-off MOSFET. R0 is the current limiting resistor. V2 is the adjustable voltage.

The pulse width, pulse period and phase sequence difference of the SCM can be adjusted by the upper computer, and the voltage waveform on R0 can be measured. The results of the experiment are shown in Fig. 5. It can be seen from this figure that the upper computer can adjust the pulse generator accurately.

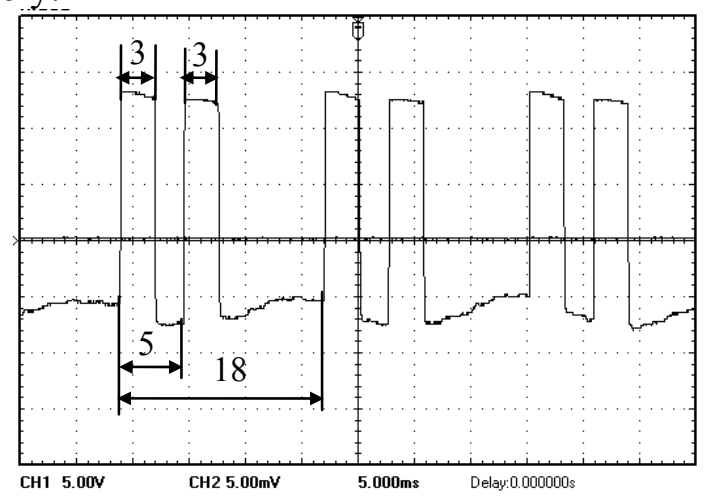

(a)

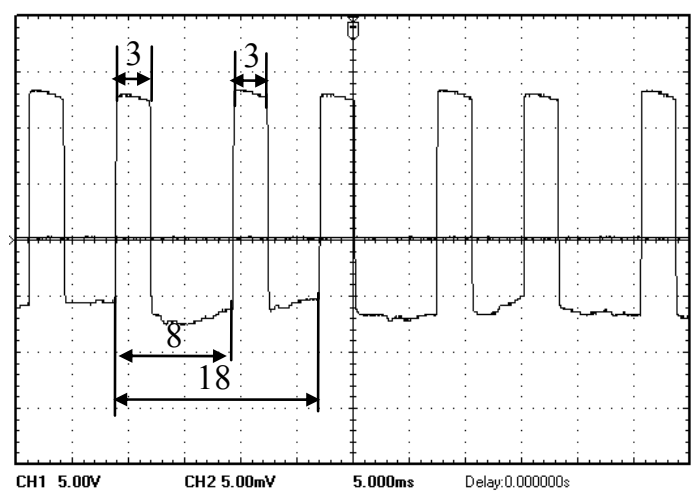

(b)

Fig. 5 Voltage waveform of the current-limiting resistor with different pulse parameter settings: (a) pulse width of $3 \mathrm{~ms}$, pulse period of $18 \mathrm{~ms}$, phase sequence difference of $5 \mathrm{~ms}$ (b) pulse width of $3 \mathrm{~ms}$, pulse period of $18 \mathrm{~ms}$, phase sequence difference of $8 \mathrm{~ms}$.

\section{Conclusions}

The multi-channel PWM signals are developed based on the AT89C52 SCM in this paper. The pulse width, pulse period and phase sequence difference can be adjusted by the upper computer at a wide range. VB is used to program the controlling interface of the pulse generator, the simulation and serial communication with SCM are achieved, and the pulse width, pulse period, and phase sequence difference can be randomly adjusted conveniently. The simulation experiment results show that the pulse generator can be adjusted accurately by the upper computer.

\section{Acknowledgements}

The work is partially supported by a grant from the National Natural Science Foundation of China (Grant No. 51205411), a grant from Shandong Provincial Natural Science Foundation of China (Grant No. ZR2012EEL15), a grant from the Fundamental Research Funds for the Central Universities (Grant No. 11CX04031A), a grant from Taishan Scholar project of Shandong Province (TS20110823), and a grant from Science and Technology Development Project of Shandong Province (2011GHY11520).

\section{References}

[1] Y.F. Chen, Y.J. Lin, Y.C. Lin, S.L. Chen and L.R. Hsu: Proc Inst Mech Eng Part B J Eng Manuf Vol. 224 (2010), p. 195

[2] S. Clijsters, K. Liu, D. Reynaerts and B. Lauwers: J Mater Process Technol Vol. 210 (2010), p. 631

[3] N. Mohd Abbas, D.G. Solomon and M. Fuad Bahari: Int J Mach Tools Manuf Vol. 47 (2007), p. 1214

[4] J.C. Liu, J.C. Bai and Y.F. Guo: Non-traditional Machining (China Machine Press, China 2009) 